\title{
Ultrastable optical frequency dissemination on a multi-access fibre network
}

\author{
Anthony Bercy ${ }^{1,2} \cdot$ Olivier Lopez $^{1} \cdot$ Paul-Eric Pottie $^{2} \cdot$ Anne Amy-Klein $^{1}$
}

Received: 6 March 2016 / Accepted: 6 June 2016 / Published online: 25 June 2016

(C) The Author(s) 2016. This article is published with open access at Springerlink.com

\begin{abstract}
We report a laboratory demonstration of the dissemination of an ultrastable optical frequency signal to two distant users simultaneously using a branching network. The ultrastable signal is extracted along a main fibre link; it is optically tracked by a narrow linewidth laser diode, which light is injected in a secondary link. The propagation noise of both links is actively compensated. We implement this scheme with two links of 50-km fibre spools, the extraction being set up at the mid-point of the main link. We show that the extracted signal at the end of the secondary link exhibits a fractional frequency instability of $1.4 \times 10^{-15}$ at 1 -s measurement time, almost equal to the $1.3 \times 10^{-15}$ instability of the main link output end. The long-term instabilities are also very similar, at a level of $3-5 \times 10^{-20}$ at $3 \times 10^{4}$-s integration time. We also show that the setting up of this extraction device, or of a simpler one, at the main link input, can test the proper functioning of the noise rejection on this main link. This work is a significant step towards a robust and flexible ultrastable network for multi-users dissemination.
\end{abstract}

Anne Amy-Klein

amy@univ-paris13.fr

1 Laboratoire de Physique des Lasers, Université Paris 13, Sorbonne Paris Cité, CNRS, 99 Avenue Jean-Baptiste Clément, 93430 Villetaneuse, France

2 Laboratoire National de Métrologie et d'Essais-Système de Références Temps-Espace, Observatoire de Paris, PSL Research University, CNRS, Sorbonne Universités, UPMC Univ. Paris 06, 61 Avenue de l'Observatoire, 75014 Paris, France

\section{Introduction}

Optical fibre links enable us to transfer an ultrastable frequency between distant laboratories with almost no degradation [1,2]. Since 10 years, they have been extended from a few hundreds of kilometre to a record length of $1840-\mathrm{km}$ [3] and noise compensation has been improved leading to an accuracy of the frequency transfer in the $10^{-20}$ range [4] It is the best mean for comparing distant optical clocks as recently demonstrated on a $1415-\mathrm{km}$ fibre distance between the French and German National Metrological Institutes in Paris and Braunschweig [5]. Behind fundamental frequency metrology, optical links open new applications such as high-precision measurements of atomic or molecular absolute frequencies [6-9], or frequency dissemination to radio antenna in radio astromy [10].

However, the development of optical links is now slowed down by the difficulty and cost to access the fibres. Even if optical links were also implemented on active telecommunication fibres [11, 12], using wavelength division multiplexing, a smart management of the fibre network is required in order to extend the dissemination to many laboratories. In that perspective, a point-to-point distribution requires too many fibres and a branching network is much more effective. It was first proposed and demonstrated to extract the ultrastable signal for multiple users along a main link [13-16]. Another possibility consists in implementing a branching optical fibre network with noise correction at each output end [17]. In this paper, following first proposal for radio frequency and optical frequency multi-user dissemination $[13,15]$, we demonstrate the implementation of a secondary link, injected with a laser diode phase-locked to the signal extracted on a main optical link. This new architecture enables us to disseminate an ultrastable signal 
from one laboratory to many distant users, who are not necessarily along the main link. This is especially suitable for multi-users dissemination in a metropolitan area network.

In this paper, we will first explain the principle of multiuser dissemination using extraction and a secondary link. We will then describe the experimental set-up based on a main and a secondary link of 50-km fibre spools. We will show the experimental results for the instability and the accuracy of the extracted signal, which is copying the input signal with a very high fidelity. Finally, we will show that such a device can be used to test the proper functioning of the main link, as an alternative to the usual end-to-end measurement.

\section{Principle}

Figure 1 shows the principle of the multiple dissemination to distant users from a main link, first proposed in [15] for radio frequencies and in [13, 14] for optical frequencies. For clarity, we will first consider the distribution of an ultrastable signal to two users Out0 and Out1. A noise-compensated optical link is established to Out0 user, using the well-known technique of the round-trip noise compensation [1, 2]. Corrections are implemented using an acousto-optic modulator ( $\mathrm{AOM})$ at the link input end. Instead of establishing a second optical link to Out1 user, part of the main link is used to disseminate the signal to an intermediate point, called extraction point, where a coupler is inserted to extract both forward and backward signals propagating in the main link, of frequency $v_{+}$and $v_{-}$, respectively [13-16]. As already proposed in [13], a narrow bandwidth laser diode is offset phase-locked to the forward extracted signal and feeds a secondary optical link to Out1. This secondary link is also noise compensated, using the same method as for the main link. Other secondary links can be fed by the laser diode which enables us to distribute the ultrastable signal to many users simultaneously. A second laser diode can also be phase-locked to the backward extracted signal and feed other secondary links. In that case, the noise compensation will use acousto-optics modulator of opposite shift compared to the ones used for the links fed by the first laser diode [18].

This scheme includes an extraction stage along the main link, as initially proposed in [16] and demonstrated in $[13-15,18]$. Both forward and backward extracted signals exhibit phase fluctuations, because the noise correction at the main link input is overcorrecting the phase noise at this intermediate point. Let us consider that the extraction is performed at a distance $L_{\mathrm{A}}$ from the input end, with the integrated phase noise $\phi_{\mathrm{A}}$, and $L_{\mathrm{B}}$ from the output end, with the integrated phase noise $\phi_{\mathrm{B}}$ (see Fig. 1). The phase fluctuations on the forward extracted signal are given by the sum of the noise $\phi_{\mathrm{A}}$ and the main link correction signal $\phi_{\mathrm{C} 0}=-\left(\phi_{\mathrm{A}}+\phi_{\mathrm{B}}\right)$, which results in a noise $-\phi_{\mathrm{B}}$. To compensate it, we detect the beatnote of the two extracted signals, which exhibits phase fluctuations $2 \phi_{\mathrm{B}}$. The latter signal, after division by two, is mixed with the control signal of the laser diode, which phase fluctuations are thus free of the main link phase noise.

This set-up enables us to distribute the ultrastable signal to many remote users simultaneously along a main link. It is compatible with optical amplification on main or secondary link and thus with distribution to sites more than $100 \mathrm{~km}$ away. Its implementation can be fully automatised and does not require the local RF oscillator to be stable and
Fig. 1 Principle of the multiuser distribution; a laser diode is phase-locked onto the ultrastable signal extracted along the main link and feeds secondary links, which noise is compensated; OC, optical coupler; LO, local oscillator; $\mathrm{PC}$, polarisation controller

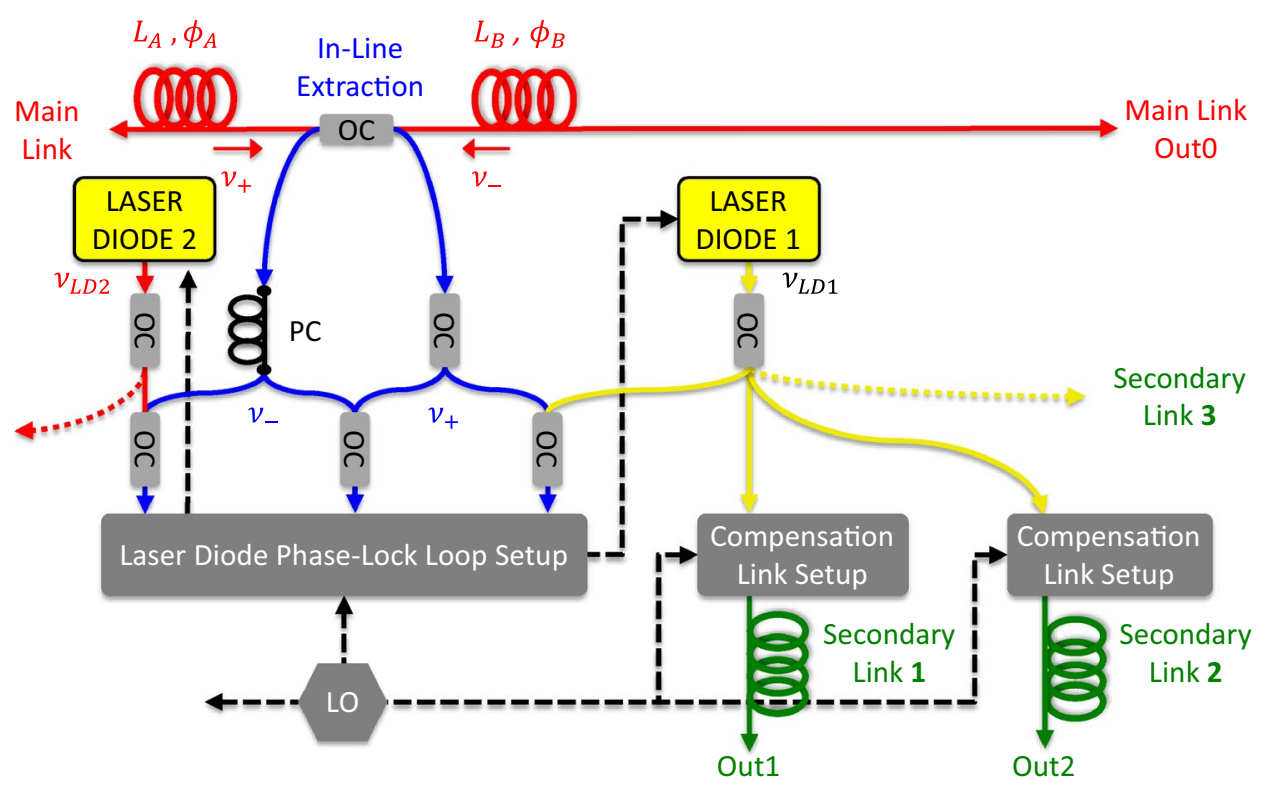


accurate. It is very flexible, since one additional extraction coupler and/or one additional secondary link can be set up on the main link without changing the overall dissemination architecture. In contrast to the branching architecture proposed in [17], the main link is fully independent of the secondary links. However, the noise rejection at the secondary links output depends on the proper functioning of the main link.

\section{Experimental set-up description}

The experimental set-up was already introduced in [13] and is shown in Fig. 2. It is composed of three parts, the extraction set-up, the optical regeneration with the laser diode, and the noise compensation of the secondary link, respectively, labelled 1-3 in Fig. 2.

The main link is set-up with the usual round-trip noise compensation method $[1,11]$, corrections being applied at the link input end through an AOM (AOM1) of frequency $f_{1}$. It is fed with an ultrastable laser of frequency $v_{0}$ disseminated from SYRTE to LPL through a free-running optical link of $43 \mathrm{~km}$, leading to frequency instabilities in the range of $10^{-14}$ [19]. These instabilities are low enough to test optical links of lengths up to a few hundreds of kilometre.
Part of the forward and backward signals propagating on the main link are extracted using a coupler and recombined using a second coupler (part 1 in blue in Fig. 2). This Mach-Zehnder-type interferometer gives a beatnote signal on a first photodiode PD1 at frequency $2 \times f_{2}$, where $f_{2}$ is the frequency of the AOM at the main link output. This signal exhibits the noise $2 \phi_{\mathrm{B}}$ of the upstream part of the main link. We insert a polarisation controller to align the polarisations of the two beams, which are perpendicular at the extraction coupler output, since a Faraday mirror is used to reflect the signal at the end of the main link. A $90^{\circ}$ Faraday rotator could also be used.

The beatnote signal between the laser diode and the forward extracted signal is detected with a second photodiode PD2 (part 2 in yellow in Fig. 2). A polarisation controller at the laser diode output (not shown in Fig. 2) enables us to optimise its amplitude. This beatnote signal exhibits both the laser diode phase fluctuations $\phi_{\mathrm{LD}}$ and the main link propagation noise $-\phi_{\mathrm{B}}$. The latter noise is rejected by mixing this beatnote signal with half the beatnote signal detected on PD1. The resulting signal is used to phase-lock the laser diode with an offset frequency $f_{\mathrm{LD}}$ given by the local oscillator frequency $f_{\mathrm{LO}}: f_{\mathrm{LD}}=f_{\mathrm{LO}}$. The corrections are applied to both the laser temperature and current, with a bandwidth of approximately $100 \mathrm{kHz}$. The laser diode frequency is thus $v_{\mathrm{LD}}=v_{0}+f_{1}+f_{2}+f_{\mathrm{LO}}+\dot{\phi}_{\mathrm{LO}} / 2 \pi$, where

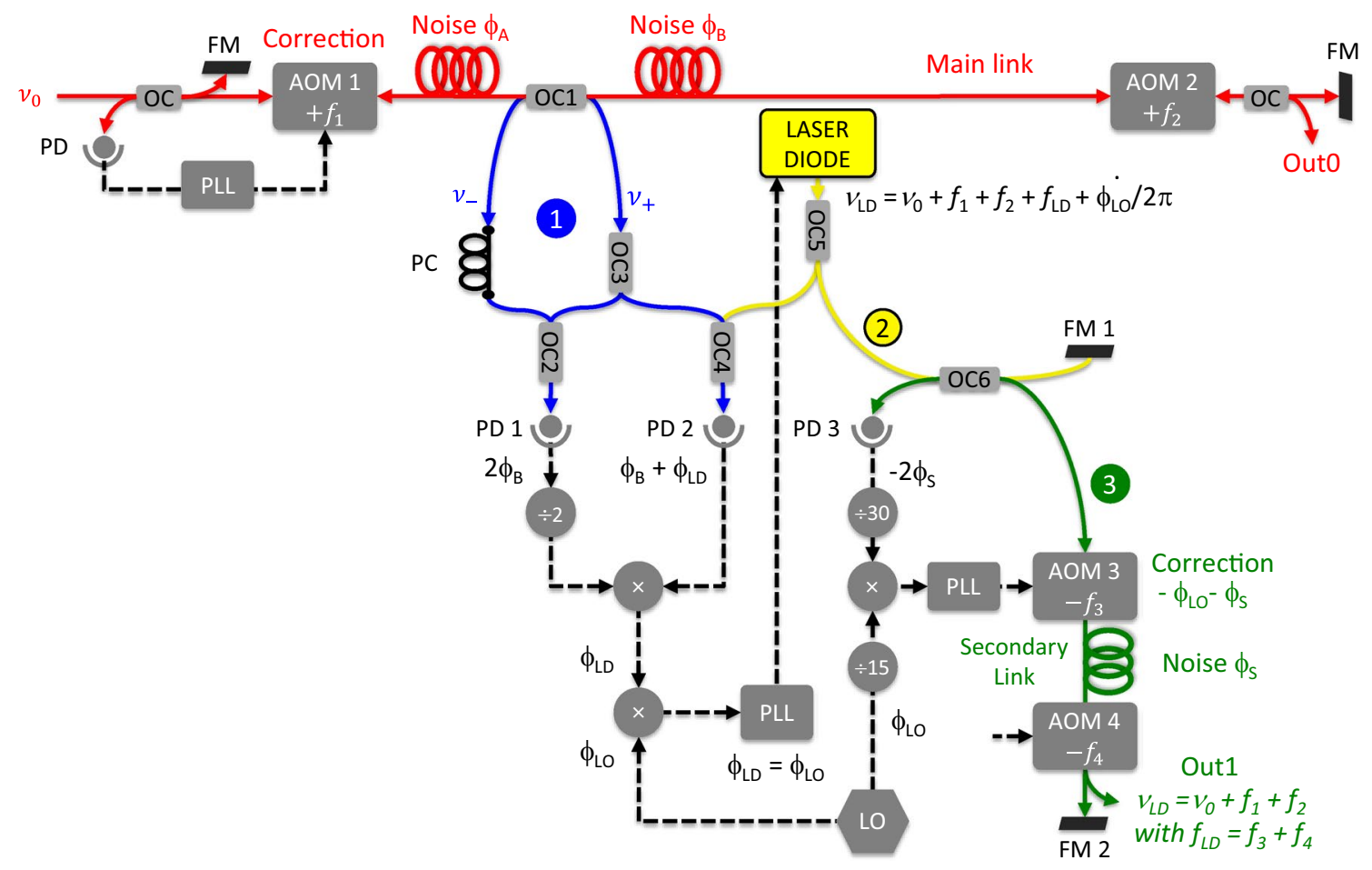

Fig. 2 Set-up for a secondary link with noise compensation; OC, optical coupler; AOM, acousto-optic modulator; PLL, phase-lock loop; FM, Faraday mirror; PD, photodiode; LO, local oscillator; PC, polarisation controller. For simplicity, only the phases have been indicated for the PLLs 
the phase fluctuations are copying the phase fluctuation $\phi_{\mathrm{LO}}$ of the local oscillator (within the loop bandwidth).

The laser diode is feeding the secondary optical link which noise $\phi_{S}$ is compensated using the round-trip method, similarly to the main link (part 3 in green in Fig. 2). Two AOMs (denoted AOM3 and AOM4) of negative frequency shifts $f_{3}$ and $f_{4}$ are used at the input and output of this secondary link, respectively. The beatnote between the round-trip signal and the input signal, of frequency $2 \times\left(f_{3}+f_{4}\right)$, is detected through a Michelson-type interferometer. It exhibits the phase noise $-2 \phi_{\mathrm{S}}$ (the minus sign arises from the negative frequency shifts). After division by 30 , it is mixed with the signal of the local oscillator, itself divided by 15 , in order to generate corrections applied through AOM3, with a frequency $f_{3}=f_{\mathrm{LO}}-f_{4}$ and a phase $\phi_{\mathrm{C} 1}=-\left(\phi_{\mathrm{S}}+\phi_{\mathrm{LO}}\right)$. The division factors 30 and 15 were chosen in order that the error signal is much less than $1 \mathrm{rad}$, so that a cycle slip of the PLL has a very low probability (typically less than $10^{-4}$ in our set-up). Their ratio should be exactly 2 in order that half the round-trip link noise is corrected, with corrections applied to AOM 3. As a result, the secondary link output frequency is copying the ultrastable frequency at the main link output $v_{0}+f_{1}+f_{2}$ without any added phase noise.

Although using a local RF oscillator for the phase-lock loops and AOM drivers, this set-up is insensitive to its frequency fluctuations. We have indeed engineered the set-up so that the RF oscillator instabilities and bias added with the laser diode phase-lock loop are exactly compensated by the secondary link phase corrections. This passive noise compensation is similar to the one implemented in the laser stations we developed for cascaded optical links [12].

Furthermore, we have designed this set-up in order to minimise the noise resulting from uncompensated fibre paths [13]. The fibre lengths are minimised, and the whole passive optical set-up (including the couplers and interferometers) is very compact and installed in an aluminium box which is actively temperature-stabilised at $30{ }^{\circ} \mathrm{C}$ and surrounded with insulating foam. Moreover, we carefully adjusted the fibre lengths so that the temperature sensitivity of the set-up is further reduced. We chose the fibre lengths of both arms between the couplers OC1 and OC2 to be identical (see Fig. 2). In the case of a homogeneous temperature, the phase variation due to any temperature change will thus cancel between the two arms. We also set the fibre length between the laser diode and the optical coupler OC4 to be the sum of the fibre lengths between OC1 and OC4 and between the laser diode output and the Faraday mirror FM1 after OC6. This way, we add to the laser diode phase the opposite of the uncompensated fibre phase variation between the laser output and the Faraday mirror FM1 and the resulting total phase variation cancels.

\section{Results and discussion}

We implement the multi-user distribution scheme of Fig. 2 at the mid-point of an optical link of 50-km fibre spools. The secondary link is $50 \mathrm{~km}$ long. We use an interferometric setup to detect simultaneously the beatnotes between the input end of the link and the output ends of the main and secondary links, respectively, labelled Out0 and Out1 in Fig. 2. These beatnotes enable us to characterise the performance of the frequency transfer at the main and secondary links output ends, the latter being denoted by extraction output below.

The phase noise power spectral densities (PSD) of these two signals are plotted in Fig. 3 [red curve (c) and green curve (d), respectively]. The phase noise PSD of the freerunning main link [orange curve (a)] and extraction output [black curve (b)] are also shown. In contrast to the other curves, the latter were measured with a frequency voltage converter which limits the measurement sensitivity after $1 \mathrm{kHz}$. Both curves are very similar and typical for optical fibre links, with a noise of approximately $10 \mathrm{rad}^{2} /$ $\mathrm{Hz}$ at $1 \mathrm{~Hz}$ and $10^{-6} \mathrm{rad}^{2} / \mathrm{Hz}$ at $1 \mathrm{kHz}$. Both compensated noises are also very similar with a phase noise PSD below $10^{-5} \mathrm{rad}^{2} / \mathrm{Hz}$ between 1 and $50 \mathrm{~Hz}$. Noise is corrected up to about $150 \mathrm{~Hz}$, with a correction overshoot of a few hundreds of $\mathrm{Hz}$ compatible with the theoretical bandwidth of $1 \mathrm{kHz}$ given by $1 / 4 \tau$ with $\tau$ the propagation delay [2]. This limit is the same for both links but, in case of different lengths, the bandwidth of the extraction is limited by the longer delay [13]. We checked that the noise floors of both outputs are below these PSDs. The noise rejection of around $10^{6}$ at $1 \mathrm{~Hz}$ is also compatible with the theoretical limit given by $\frac{1}{3}(2 \pi f \tau)^{2}$ which is $8 \times 10^{7}$ for a $50-\mathrm{km} \mathrm{link}$ [2]. This shows that the noise rejection is optimised.

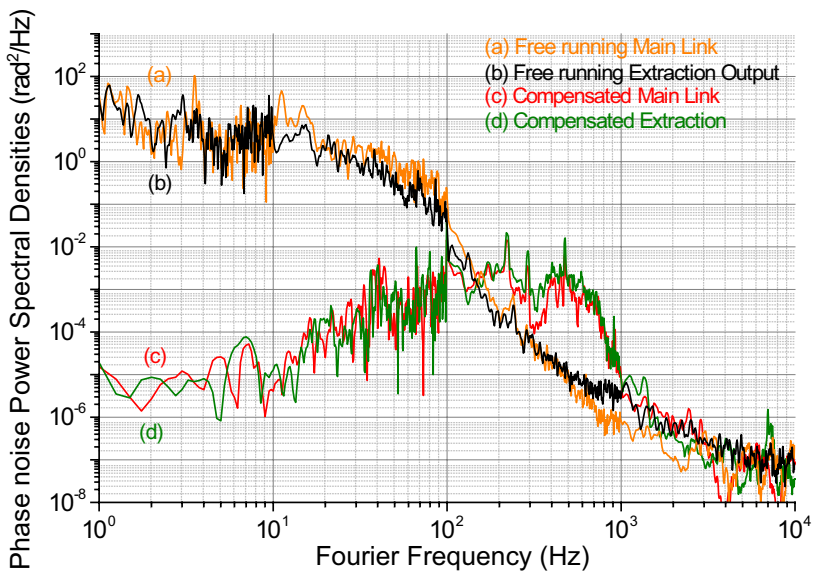

Fig. 3 Phase noise power spectral density of $a$ the free-running main link (orange line), $b$ the free-running extraction output (black line), $c$ the compensated main link (red line), and $d$ the compensated extraction output (green line) 


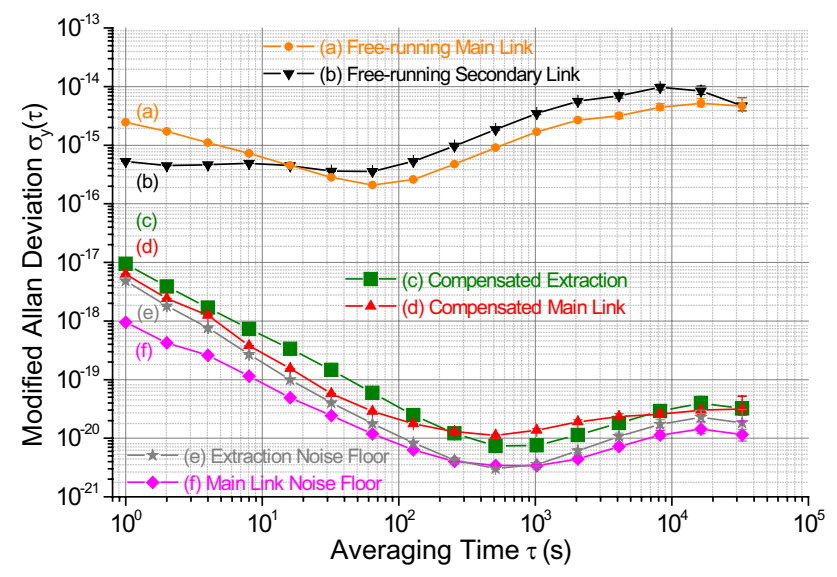

Fig. 4 End-to-end fractional frequency instability, calculated from $\Lambda$-type data with the Modified Allan deviation, of $a$ the $50-\mathrm{km}$ freerunning main link (orange circles), $b$ the $50-\mathrm{km}$ secondary link (black down-triangles), $c$ the compensated extraction output (green squares), $d$ the compensated main link output (red up-triangles), $e$ the extraction noise floor (grey stars), and $f$ the main link noise floor (pink diamonds)

To evaluate the end-to-end stabilities, the beatnotes at frequency $f_{1}+f_{2}=75 \mathrm{MHz}$ are tracked with a 100$\mathrm{kHz}$ bandwidth, and after frequency division by 2 they are recorded simultaneously with a dead-time free counter (Kramer + Klische FXE) with a gate time of $1 \mathrm{~s}$ and $\Lambda$-type operation, in order to average out the noise in a narrow bandwidth [20, 21]. Then, we use the modified Allan deviation to characterise the stability of the extraction set-up.

Figure 4 displays the experimental stability of the free-running main link [orange circles (a)] and secondary link [black down-triangles (b)]. Their noises slightly differ because the main link fibre is wound onto a spool and thus experiences some constraints, whereas the secondary link fibre is wound freely (without any central spool). These noises are quite low compared to that of installed telecommunication fibres, leading to a very low instability of the extraction and main link outputs at short integration time. The extraction output stability [green squares (c)] is $1 \times 10^{-17}$ at 1 -s averaging time, decreases and reaches a floor of approximately $4 \times 10^{-20}$ after $10^{4} \mathrm{~s}$. It is at the state of the art for fibre frequency transfer on such length of fibre spools and nearly copies the main link output stability [red up-triangles (d)]. Note that the noise correction is very robust and that the set-up was operated during 2.5 day without any cycle slip. At short term, the stability is slightly above the main link output stability which is $6 \times 10^{-18}$ at 1 -s averaging time. The extraction output indeed exhibits both the contribution of the signal extracted from the main link (after compensation of $\phi_{\mathrm{B}}$ ) and the residual noise of the secondary link, the latter

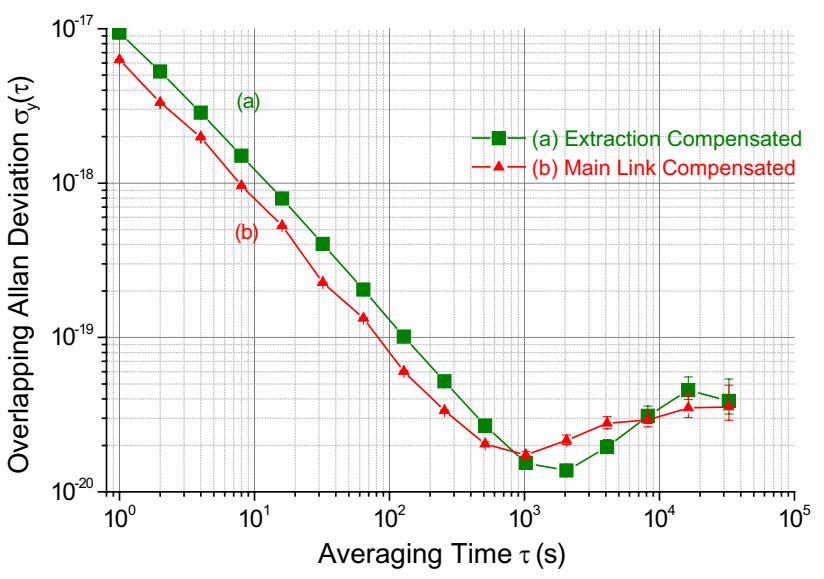

Fig. 5 End-to-end fractional frequency instability, calculated with the overlapping Allan deviation from the same $\Lambda$-type data as used for Fig. 4, of $a$ the compensated extraction output (green squares) and $b$ the compensated main link output (red up-triangles)

limiting the noise rejection at short term. Figure 4 also shows the noise floors for both the main link [pink diamonds (f)] and the extraction output [grey stars (e)], which limit the long-term stability. The latter is mainly attributed to thermal noise on uncompensated fibre paths, due to imperfect length adjustment and thermal stabilisation in the extraction optical set-up, or in the interferometric measurement set-up.

Finally, the accuracy of the frequency transfer was evaluated by calculating the mean value of the end-to-end beatnote frequency offset. Following [4, 22], we estimate its statistical fractional uncertainty as the long-term overlapping Allan deviation at 20,000 or $30,000 \mathrm{~s}$ of the data set. As shown in Fig. 5, we obtained for the main link a mean frequency offset of $-8.2 \times 10^{-21}$ with a statistical uncertainty of $4 \times 10^{-20}$. For the extraction output, the mean frequency offset is $-2.4 \times 10^{-21}$ with a statistical uncertainty of $4 \times 10^{-20}$. These figures show that the frequency transfer exhibits no bias to the expected value.

With this link performance, the ultrastable laser is thus transferred through the secondary and main links without degradation. Even an ultrastable laser exhibiting a record $10^{-16}$ stability at 1 -s averaging time [23] would be faithfully transferred for integration time above $1 \mathrm{~s}$.

\section{Testing the noise rejection on the main link}

We have shown that our extraction set-up enables us to transfer the ultrastable signal to several users simultaneously, using secondary links to reach the more distant ones. We are now discussing an alternative application of an extraction set-up, which consists in testing the proper functioning of the noise rejection on the main link. 
As pointed out by several authors, one can test the performance of an optical link by analysing the end-to-end stability, which requires that the two ends are at the same place. Thus, optical links are usually implemented with two parallel fibres or with a loop fibre. But it is not always possible to get such configuration, which requires the doubling of the fibre lengths to the distant lab, and thus increases the equipment and maintenance costs. Calonico and co-workers implemented a double round trip on a single fibre in order to characterise the link performance [24]. This alternative method is very beneficial for long-haul link network development, but could be difficult to implement when the fibre attenuation and parasitic noise are large. Here, we propose to use an extraction set-up at the link input to test the good functioning of the noise rejection on the main link. As demonstrated in [13], the extraction end phase noise PSD without any secondary link is lower or equal to the main link output phase noise PSD. When the extraction occurs at the link input, the phase correction at the extraction output is exactly opposite to the roundtrip fibre phase noise and thus to the correction at the link input. Therefore, following our simple model detailed in [13], the extracted signal copies exactly the input signal and no fibre phase fluctuation is added to the signal. Thus, by analysing the extracted signal, we can infer that the noise correction signal applied at the link input is properly compensating the main link noise. For that purpose, one has to carefully implement the stability measurement set-up: the lengths of the fibres used to detect the beatnote between the input end of the link and the extraction end should be carefully balanced and thermalised so that the noise arising from these uncommon fibre paths is negligible.

In order to validate this method, we implemented the extraction set-up at the main link input, and we replaced the secondary link with an equivalent attenuator. Then, we compare the main link output stability with the extraction stability for the following three cases: the free-running main link, a partial noise compensation of the main link, with a poor adjustment of the loop gains, and an optimised noise compensation of the main link. The three pairs of stabilities are shown in Fig. 6, with red up-triangles for the main link and green squares for the extraction output. In each case, the extraction stability is almost copying the main link output stability. Note that for the compensated main link, the extraction stability is $5.9 \times 10^{-18}$ at 1 -s averaging time when the main link output stability is $7.5 \times 10^{-18}$. The ratio of the squares of these stabilities gives an estimate of the F-factor we introduced in [13]. It is 0.6 , when the theoretically expected value is 0 . This can be explained by the fact that the stability at $1 \mathrm{~s}$ is limited by the noise floor (as shown in Fig. 4) which is expected to be mainly due to the electronic detection set-up.

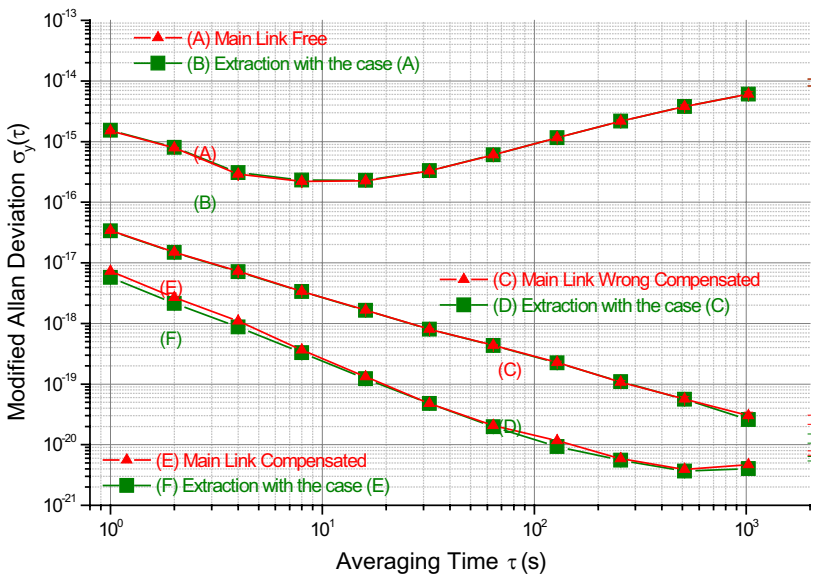

Fig. 6 End-to-end fractional frequency instability, calculated from $\Lambda$-type data with the Modified Allan deviation, of the main link (red up-triangles) and the extraction output (green squares) for three pairs of data: $a, b$ free-running main link, $c, d$ poorly compensated main link, and $e, f$ compensated main link. Error bars are too small to show in figure

The stabilities displayed in Fig. 6 show that the extraction stability is a very good measurement of the main link stability and enables us to check the right functioning of the noise rejection. Such set-up can be used to test the proper operation of a single-fibre link. This is very beneficial when implementing the first spans of a cascaded link without having set-up the next spans. This is also very useful when implementing an uncompensated link to a distant user, in order to evaluate these user link instabilities. Note that, for these applications, we do not need to phase-lock a laser diode to the extracted signal; the simpler extraction set-up demonstrated in [13] is more than enough.

\section{Conclusion}

We have demonstrated the distribution of an ultrastable frequency to two distant users using a single-link input. We use a new type of branching network topology with a secondary link departing from any position along a main link. This architecture enables us to establish an ultrastable frequency distribution to multiple users, for example in a dense metropolitan area. It is a very interesting alternative to point-topoint distribution, which requires one optical link per user and thus a higher cost of fibre equipment and maintenance. We have shown that the stability at the secondary link output is nearly copying that at the main link output, with an Allan deviation of $10^{-17}$ at 1-s averaging time. This set-up is very robust and can be operated without any stable RF oscillator at the extraction point, since the secondary link output stability is independent of this local oscillator's instabilities. It can be implemented on an active telecommunication 
network with simultaneous data traffic, provided that we set-up optical add-drop multiplexers to insert or extract the ultrastable signal from the data traffic [11].

This work is a major step of the French project named REFIMEVE + [25]. REFIMEVE + is developing a wide national infrastructure where a reference optical signal generated at SYRTE will be distributed to about 20 academic and institutional users using the national academic network RENATER. Such an extraction set-up will be mainly implemented for metropolitan area distribution, as for example in the Paris area. It is also a key element of the current effort to establish continental ultrastable fibre network. Multi-users dissemination opens the way to a wide distribution of an ultrastable frequency reference, enabling applications beyond metrology [6-10], and we expect that it will stimulate the development of new high-sensitivity experiments in a broad range of applications.

Acknowledgments We deeply acknowledge Giorgio Santarelli for fruitful discussions and Christian Chardonnet and Saïda GuellatiKhelifa for stimulating this work. We also acknowledge A. Kaladjian and F. Wiotte from LPL for technical support. We acknowledge funding support from the Agence Nationale de la Recherche the Agence Nationale de la Recherche (ANR blanc LIOM 2011-BS04-009-01, Labex First-TF ANR 10 LABX 48 01) and IFRAF-Conseil Régional Ile-de-France. Results incorporated in this standard received funding from the EMPIR programme co-financed by the Participating States and from the European Union's Horizon 2020 research and innovation programme.

Open Access This article is distributed under the terms of the Creative Commons Attribution 4.0 International License (http://creativecommons.org/licenses/by/4.0/), which permits unrestricted use, distribution, and reproduction in any medium, provided you give appropriate credit to the original author(s) and the source, provide a link to the Creative Commons license, and indicate if changes were made.

\section{References}

1. L.S. Ma et al., Delivering the same optical frequency at two places: accurate cancellation of phase noise introduced by an optical fiber or other time-varying path. Opt. Lett. 19(21), 17771779 (1994)

2. N.R. Newbury, P.A. Williams, W.C. Swann, Coherent transfer of an optical carrier over $251 \mathrm{~km}$. Opt. Lett. 32(21), 3056 (2007)

3. S. Droste et al., Optical-frequency transfer over a single-span 1840 km fiber link. Phys. Rev. Lett. 111(11), 110801 (2013)

4. S.M.F. Raupach, A. Koczwara, G. Grosche, Brillouin amplification supports $1 \times 10-20$ accuracy in optical frequency transfer over $1400 \mathrm{~km}$ of underground fibre. Phys. Rev. A 92(2), 021801(R) (2015)

5. C. Lisdat, G. Grosche, N. Quintin, C. Shi, S.M.F. Raupach, C. Grebing, D. Nicolodi, F. Stefani, A. Al-Masoudi, S. Dörscher, S. Häfner, J.-L. Robyr, N. Chiodo, S. Bilicki, E. Bookjans, A. Koczwara, S. Koke, A. Kuhl, F. Wiotte, F. Meynadier, E. Camisard,
M. Abgrall, M. Lours, T. Legero, H. Schnatz, U. Sterr, H. Denker, C. Chardonnet, Y. Le Coq, G. Santarelli, A. Amy-Klein, R. Le Targat, J. Lodewyck, O. Lopez, P.-E. Pottie, A new era of international clock measurements (2015). arXiv:1511.07735

6. A. Matveev et al., Precision measurement of the hydrogen 1S-2S frequency via a 920-km fiber link. Phys. Rev. Lett. 110(23), 230801 (2013)

7. C. Clivati et al., Measuring absolute frequencies beyond the GPS limit via long-haul optical frequency dissemination. Opt. Express 24(11), 11865-11875 (2016)

8. B. Chanteau et al., Mid-infrared laser phase-locking to a remote near-infrared frequency reference for high-precision molecular spectroscopy. New J. Phys. 15(7), 073003 (2013)

9. B. Argence et al., Quantum cascade laser frequency stabilization at the sub-Hz level. Nat. Photonics 9(7), 456-460 (2015)

10. C. Clivati, G.A. Costanzo, M. Frittelli, F. Levi, A. Mura, M. Zucco, R. Ambrosini, C. Bortolotti, F. Perini, M. Roma, D. Calonico, A coherent fiber link for very long baseline interferometry. IEEE Trans. Ultrason. Ferroelectr. Freq. Control 62(11), 1907-1912 (2015)

11. O. Lopez et al., Cascaded multiplexed optical link on a telecommunication network for frequency dissemination. Opt. Express 18(16), 16849 (2010)

12. N. Chiodo et al., Cascaded optical fiber link using the internet network for remote clocks comparison. Opt. Express 23(26), 33927-33937 (2015)

13. A. Bercy et al., In-line extraction of an ultrastable frequency signal over an optical fiber link. J. Opt. Soc. Am. B 31(4), 678-685 (2014)

14. G. Grosche, Eavesdropping time and frequency: phase noise cancellation along a time-varying path, such as an optical fiber. Opt. Lett. 39(9), 2545-2548 (2014)

15. P. Krehlik et al., Multipoint dissemination of RF frequency in fiber optic link with stabilized propagation delay. IEEE Trans. Ultrason. Ferroelectr. Freq. Control 60(9), 1804-1810 (2013)

16. G. Grosche, Method for making available a reference frequency. Ger. Pat. Appl. DE Patent 10.2008.062.139 (2010)

17. S.W. Schediwy et al., High-precision optical-frequency dissemination on branching optical-fiber networks. Opt. Lett. 38(15), 2893-2896 (2013)

18. Y. Bai et al., Fiber-based multiple-access optical frequency dissemination. Opt. Lett. 38(17), 3333-3335 (2013)

19. H. Jiang et al., Long-distance frequency transfer over an urban fiber link using optical phase stabilization. J. Opt. Soc. Am. B 25(12), 2029-2035 (2008)

20. E. Rubiola, On the measurement of frequency and of its sample variance with high-resolution counters. Rev. Sci. Instrum. 76, 054703 (2005)

21. S.T. Dawkins, J.J. McFerran, A.N. Luiten, Considerations on the measurement of the stability of oscillators with frequency counters. IEEE Trans. Ultrason. Ferroelectr. Freq. Control 54, 918925 (2007)

22. O. Lopez et al., Frequency and time transfer for metrology and beyond using telecommunication network fibres. Comptes Rendus Phys. 16, 531-539 (2015)

23. T. Kessler et al., A sub-40-mHz-linewidth laser based on a silicon single-crystal optical cavity. Nat. Photonics 6(10), 687-692 (2012)

24. D. Calonico et al., High-accuracy coherent optical frequency transfer over a doubled 642-km fiber link. Appl. Phys. B 117(3), 979-986 (2014)

25. Refimeve+, project www.refimeve.fr/ 